\title{
Causal explanation improves judgment under uncertainty, but rarely in a Bayesian way
}

\author{
Brett K. Hayes $^{1} \cdot$ Jeremy Ngo $^{1}$ - Guy E. Hawkins ${ }^{2} \cdot$ Ben R. Newell ${ }^{1}$
}

Published online: 13 September 2017

(C) Psychonomic Society, Inc. 2017

\begin{abstract}
Three studies reexamined the claim that clarifying the causal origin of key statistics can increase normative performance on Bayesian problems involving judgment under uncertainty. Experiments 1 and 2 found that causal explanation did not increase the rate of normative solutions. However, certain types of causal explanation did lead to a reduction in the magnitude of errors in probability estimation. This effect was most pronounced when problem statistics were expressed in percentage formats. Experiment 3 used process-tracing methods to examine the impact of causal explanation of false positives on solution strategies. Changes in probability estimation following causal explanation were the result of a mixture of individual reasoning strategies, including nonBayesian mechanisms, such as increased attention to explained statistics and approximations of subcomponents of Bayes' rule. The results show that although causal explanation of statistics can affect the way that a problem is mentally represented, this does not necessarily lead to an increased rate of normative responding.
\end{abstract}

Keywords Judgment $\cdot$ Intuitive statistics $\cdot$ Bayesian reasoning

Electronic supplementary material The online version of this article (https://doi.org/10.3758/s13421-017-0750-z) contains supplementary material, which is available to authorized users.

Brett K. Hayes

B.Hayes@unsw.edu.au

1 School of Psychology, University of New South Wales, Sydney, NSW 2052, Australia

2 School of Psychology, University of Newcastle, University Dr, Callaghan, NSW 2308, Australia
Causal knowledge plays a major role in cognition. Such knowledge has been shown to have profound effects on the way people learn contingencies (e.g., Waldmann, Hagmayer, \& Blaisdell, 2006), categorize (Hayes \& Rehder, 2012), reason (Fernbach, Darlow, \& Sloman, 2011), and make decisions (Hagmayer \& Sloman, 2009).

Krynski and Tenenbaum (2007) claim that causal knowledge can also increase normative performance in judgments under uncertainty that involve the estimation of event probabilities. Consider, for example, the mammogram problem shown in Table 1. The goal is to estimate the conditional probability of a target hypothesis given some observations, $p(\mathrm{H} \mid \mathrm{D})$ (e.g., the probability that a person has cancer given a positive mammogram). The statistics provided include the base rate of the target hypothesis $p(\mathrm{H})$ (e.g., the probability of cancer), the likelihood of the observed data given the hypothesis $p(\mathrm{D} \mid \mathrm{H})$ (e.g., the probability of a positive mammogram given cancer), the probability of the data given alternative hypotheses $p(\mathrm{D} \mid-\mathrm{H})$ (e.g., the probability of a positive mammogram in the absence of cancer), and the base rate of the alternatives $p(\ulcorner\mathrm{H})$. The normative solution is given by Bayes' rule (Equation 1). In the Table 1 problem this leads to an estimate of $p(\mathrm{H} \mid \mathrm{D}) \approx 0.051$ (see Appendix A for the derivation).

$p(H \mid D)=\frac{p(H) \times p(D \mid H)}{p(H) \times p(D \mid H)+p(\neg H) \times p(D \mid \neg H)}$

A large body of evidence suggests that people perform poorly on such probability estimation problems (see Barbey \& Sloman, 2007, for a review). Common errors include ignoring the base rate of the target hypothesis (Evans, Handley, Perham, Over, \& Thompson, 2000; Gigerenzer \& Hoffrage, 1995; Hawkins, Hayes, Donkin, Pasqualino, \& Newell, 2015) or neglecting alternative sources of the observed data (Beyth- 
Table 1 Standard and causal judgment problems in percentage format (upper panel) and frequency format (lower panel) used in Experiment 1 (instructions in italics differed between the standard and causal versions).

\section{Mammogram problem (percentage format)}

The following statistics are known about women at age 60 who participate in a routine mammogram screening for breast cancer:

1 percent of the women have breast cancer at the time of screening.

Of those with breast cancer, $80 \%$ receive a positive result on the mammogram.

Of those without breast cancer, $15 \%$ receive a positive result on the mammogram. [Standard]

30 percent of the women without breast cancer have a benign cyst at the time of screening. Of those with a benign cyst, $50 \%$ receive a positive result on the mammogram. [Causal]

Suppose a woman gets a positive result during a routine mammogram screening. Without knowing any other symptoms, what is the probability that she has breast cancer?

\section{Mammogram problem (frequency format)}

The following statistics are known about women at age 60 who participate in a routine mammogram screening for breast cancer:

1 in every 100 women have breast cancer at the time of screening.

8 in every 10 of those with breast cancer receive a positive mammogram.

15 in every 100 women without breast cancer will get a positive mammogram. [Standard]

30 in every 100 women have a benign cyst at the time of screening. Of those with a benign cyst, half will receive a positive result on the mammogram. [Causal]

Imagine that we have selected a random sample of 100 women over 60. Without knowing any other symptoms, on average, how many women who receive a positive result during a routine mammogram screening do you expect to have breast cancer?

Marom \& Fischhoff, 1983; Hayes, Hawkins, \& Newell, 2016).

Krynski and Tenenbaum (2007) suggest that such errors reflect a failure to map the statistics given in the problem to the components of intuitive causal models. Specifically, their causal-Bayesian account suggests that there are three stages to solving judgment problems like the mammogram task. First, people construct a mental model of the causal relations between problem components. In the mammogram problem in Table 1, this involves noting that there is a generative cause (cancer) that produces the observed effect (positive mammogram). Second, they attempt to map the given statistics (e.g., base rate of cancer, probability of a positive test given cancer) to components of the mental model. Finally, people combine these components by performing Bayesian inference over their parameterized causal model to arrive at a probability estimate.

This process can fail when a key statistic (e.g., the falsepositive rate on the mammogram task) lacks a generative cause. According to Krynski and Tenenbaum (2007), people are often unsure how to incorporate such "causal orphans" into their problem representation and hence ignore or underweight them in their probability estimates. It follows that judgment performance can be improved if the causal structure of the problem is made more transparent. Krynski and Tenenbaum found that simply providing a generative explanation for false positives (e.g., they are caused by a "dense but harmless cyst that looks like a cancerous tumor") led to a substantial increase in the frequency of normative judgments ( $42 \%$ of participants given the causal version gave a normatively correct estimate, compared with just $16 \%$ in the standard version).

In sum, the causal Bayesian approach suggests that the appropriate normative standard for judgments under uncertainty involves application of Bayesian principles over specific causal models. This is a major departure from approaches based on the normative standards of classical Bayesian (e.g., Tversky \& Kahneman, 1974) or frequentist statistics (Gigerenzer \& Hoffrage, 1995). This claim is accompanied with the prospect of a relatively straightforward approach to facilitating judgment accuracy, namely, providing people with more comprehensive and detailed causal models of judgment problems.

Recent work, however, has provided mixed evidence for the causal Bayesian approach. On the positive side, there have been clear demonstrations of how the causal framing of key statistics can alter people's interpretation of judgment problems. Hayes, Hawkins, Newell, Pasqualino, and Rehder (2014), for example, found that when the false positive rate in mammogram problems had no obvious cause, it was treated as a stochastic variable, such that the observation of multiple false positives across successive tests was seen as highly unlikely. In contrast, when false positives were attributed to a specific cause (benign cyst), the probability of false positives across successive mammogram tests was seen as relatively stable.

On the negative side, two key issues remain unresolved. First, empirical work carried out since Krynski and Tenenbaum (2007) has found inconsistent evidence for the claim that causal explanation improves judgment accuracy (cf. Hayes et al., 2014; McNair \& Feeney, 2014, 2015). Second, when causal facilitation of performance has been observed, there has been no direct evidence that this is due to increased use of Bayesian reasoning.

The current studies aimed to resolve each of these issues. Experiments 1 and 2 reexamined the claim that providing causal explanation of key statistics facilitates judgment performance across a range of statistical formats. Experiment 3 involved a systematic investigation of the reasoning strategies that underlie changes in performance following provision of causal information. Specifically, we examined whether having a more detailed causal understanding of a problem leads to increased adoption of Bayesian reasoning, promotes the use of non-Bayesian heuristic strategies, or leads to a mixture of such strategies across individuals. 


\section{Does causal knowledge facilitate judgment performance?}

Studies carried out subsequent to Krynski and Tenenbaum (2007) have had difficulty replicating the strong causal facilitation effects reported in the original study. McNair and Feeney (2014) found that providing a causal explanation of false positives increased rates of normative responding on some problems, but not others. Notably, in a direct replication of the mammogram task used by Krynski and Tenenbaum (2007), they found no reliable causal facilitation of normative responding (proportion of normative responses: standard $M$ $\approx 11 \%$; causal $M \approx 23 \%$ ). McNair and Feeney (2015) also found little evidence for causal facilitation of normative responding when the effects of individual differences in arithmetic ability and cognitive ability were controlled.

Despite the negative findings concerning normative accuracy, a careful examination of the McNair and Feeney (2014, 2015) data suggests that providing a causal explanation of key statistics does affect judgment. Although causal information did not improve the rate of normatively correct answers, it did appear to reduce the magnitude of judgment errors. For example, McNair and Feeney (2014), Experiments 1-2 found that when causal explanation of false positives were given, people were less likely to make errors involving extreme overestimation of the target probability (e.g., in the mammogram problem in Table 1, estimating a probability of 0.80 or 0.65 when the normative answer was 0.05 ). In other words although causal information did not lead to an increase in exact normative estimates, it appeared to produce a closer approximation of the normative solution.

The current studies therefore reexamined the causal facilitation of performance in judgment problems involving probability estimation. Performance was measured in two ways: (a) the proportion of normatively correct responses, and (b) the magnitude of errors in probability estimation. The strong form of the causal facilitation hypothesis advanced by Krynski and Tenenbaum (2007) suggests that causal explanation should lead more people to arrive at a normatively correct Bayesian solution. The weaker form of this hypothesis is that causal explanation of key statistics may reduce estimation error even though it may not increase the number of normative responses. Experiment 1 examined these predictions with problems in which the key statistics were described as percentages as well as problems where the statistics were expressed in frequencies.

\section{What is the mechanism that drives causal facilitation?}

A deeper question is that if either form of causal facilitation of judgments is found, what is the underlying cognitive mechanism? As outlined above, Krynski and Tenenbaum (2007) make the strong claim that clearer understanding of the causal structure of a problem makes it easier to identify and integrate the components of Bayes' theorem. So far, however, this hypothesis has only been tested via predictions about improvements in the accuracy of numerical probability estimates in causal versions of judgment problems relative to standard versions. Such changes provide only indirect evidence about underlying reasoning mechanisms. The finding of some form of causal facilitation in probability estimates may be open to a variety of interpretations, some of which have little to do with enhanced Bayesian reasoning. Perhaps most obviously, providing a causal explanation may simply increase attention to relevant statistics during the judgment process, so that the reasoner is more likely to factor them into probability estimates. Such solution strategies, however, may not necessarily follow Bayesian prescriptions.

A similar argument was advanced in early work on causal influences on base-rate neglect. Ajzen (1977), for example, examined the effect of causal beliefs on use of base-rate information in a prediction task. Participants were more likely to factor base rates into predictions when there was a plausible causal connection between variables (e.g., participants had to predict the probability that an individual would pass an exam and were given the past base rate of passes). This effect was seen as the result of an attentional bias rather than an improvement in Bayesian reasoning. Supporting this conclusion, causally plausible base rates were used as a basis of prediction even when the empirical relationship between the base rate and criterion was weak (also see Bar-Hillel, 1980; Tversky \& Kahneman, 1980).

Another important issue is that different individuals may use causal information in different ways. Previous studies that have examined individual strategy use on Bayesian reasoning tasks (without additional causal information) have found that participants employ a variety of solution strategies. Gigerenzer and Hoffrage (1995), for example, examined participants' written solutions to several Bayesian problems. The protocols revealed that when statistics were presented in probability format, only a minority of participants used Bayesian reasoning (22\% of participants averaged across problems), with the proportion increasing when a frequency format was used (48\% of participants). Common non-Bayesian strategies revealed in protocol analysis included estimating the joint occurrence of the hypothesis and data, $p(\mathrm{H} \& \mathrm{D})$, confusing the likelihood, $p(\mathrm{D} \mid \mathrm{H})$, with the posterior probability, $p(\mathrm{H} \mid \mathrm{D})$, and subtraction of the false positive rate from the likelihood, $p(\mathrm{D} \mid \mathrm{H})-p(\mathrm{D} \mid \neg \mathrm{H})$. In an extensive survey of solution strategies across Bayesian problems with varying base rates, likelihoods and false positive rates, Cohen and Staub (2015) found substantial variability in which problem statistics individuals attended to and how those statistics were combined. 
Such findings highlight the complexity of the mammogram problem and suggest that both standard and causal conditions could yield a wide variety of solution strategies. Hence, Experiment 3 directly examined the reasoning mechanisms that follow causal explanation using a process-tracing approach, in which people solving judgment problems under standard or causal conditions provided verbal protocols during their problem solution. This allowed for a fine-grained test of the effects of causal information on individual solution strategies.

\section{Experiment 1}

This study examined whether causal explanation of false positives in judgment problems improves intuitive statistical estimation. In this study, we were interested in assessing the evidence for both strong facilitation (improvements in normative accuracy) and weak facilitation (reduction in error magnitude). Two statistical estimation problems were presented under standard or causal conditions. One was a mammogram problem used by Krynski and Tenenbaum (2007). The other drug-test problem had a similar general structure but used a different cover story (the goal was to estimate the probability that a person who received a positive result on a drug test had taken an illicit drug) and different statistics: $p(\mathrm{H})=0.04$, $p(\mathrm{D} \mid \mathrm{H})=0.75, p(\mathrm{D} \mid \neg \mathrm{H})=0.10$ (see Appendix B for details). In each problem, people were provided with information about the base rate of the target hypothesis (e.g., rate of cancer), the likelihood of the data conditionalized on the target hypothesis (i.e., rate of positive test findings given cancer) and the false positive rate. In the standard condition, there was no explanation of the false positive rate. In the causal condition, a brief explanation of the origin of false positives was given. The chief aim was to examine whether this additional causal information produced either strong or weak facilitation of judgment performance.

A further test of the generality of causal facilitation involved examining the effect when the relevant statistics were presented as frequencies. An important finding in studies of intuitive statistical judgment is that presenting statistics in frequency rather than a probability (or percentage) format often improves the judgment accuracy (Cosmides \& Tooby, 1996; Evans et al., 2000; Gigerenzer \& Hoffrage, 1995; Lesage, Navarrete, \& De Neys, 2013; Sirota, Juanchich, \& Hagmayer, 2014). According to the natural frequency hypothesis, this is because we are best adapted to handling computations involving observed event frequencies (Gigerenzer \& Hoffrage, 1995).

This experiment therefore examined whether causal facilitation persists in frequency-based contexts where baseline performance in statistical estimation was expected to be considerably higher than in the probability-based judgment problems used by Krynski and Tenenbaum (2007). Hence, in this study, statistical information was presented in either percentage format (e.g., " $1 \%$ of the women have breast cancer at the time of screening"), as in Krynski and Tenenbaum (2007), or in a frequency format (e.g., 1 in every 100 women had breast cancer at the time of screening; see Table 1 for further details).

This design also allowed us to examine whether causal information and frequency formats have additive or interactive effects on judgment accuracy. Krynski and Tenenbaum (2007) acknowledge that the use of frequency formats can simplify the final computation stage of probability judgment but otherwise see problems expressed in frequency formats as subject to the same causal Bayesian reasoning process as those with probabilities or percentages. This implies that switching to a frequency format may lead to a general improvement in probability estimation that would be additive with the effects of causal framing.

An alternative view is that the use of frequency formats can lead to the use of qualitatively different reasoning strategies for probability estimation (Barbey \& Sloman, 2007; Evans et al., 2000). For example, if the participant applied a common denominator to the frequencies given in Table 1 (e.g., converts them to frequencies out of 100), then the correct solution can be obtained by computing $f(\mathrm{D} \mid \mathrm{H}) / f(\mathrm{D})$, that is, $p(\mathrm{H} \mid \mathrm{D})=0.8 /$ $(0.8+15)$. Use of such a strategy would mean that there is little need to construct and apply the sort of causal model envisaged by Krynski and Tenenbaum (2007). In this case, frequency formats should lead to improved performance in both standard and causal versions of the problem, but with little additional facilitation due to causal framing.

We note that McNair and Feeney (2014, Experiment 1) compared performance on causal and standard versions of judgment problems where the statistics were expressed as frequencies. As noted earlier, causal explanation of key statistics reduced the rate of extreme errors but did not lead to increases in normative responding. Interpretation of these findings, however, is complicated by two factors. First, McNair and Feeney did not directly compare performance in percentage and frequency format versions of their judgment problems. Second, although key statistics in their problem description were presented as frequencies, participants were asked to give their estimate as a percentage. Cosmides and Tooby (1996) found that mixing statistical formats in this way reduces judgment accuracy relative to problems where both statistical descriptions and the estimation question are framed in frequentist terms. Hence, in our frequency format condition, statistics were expressed as frequencies in the problem, and a frequency-based answer was required (see Table 1). For comparison with McNair and Feeney (2014), Experiment 1 also included a mixed format condition where key statistics were presented as frequencies, but the test question requested a percentage estimate (see Appendix B for examples). 
Numerical competence has been shown to influence performance on problems involving Bayesian integration of statistics (Hill \& Brase, 2012; McNair \& Feeney, 2015; Reyna, Nelson, Han, \& Dieckmann, 2009). Hence, participants in all studies completed the Berlin Numeracy Test. This brief test provides a valid and reliable assessment of statistical numeracy and risk literacy. A particular strength is its ability to discriminate between levels of numeracy within an educated population (Cokely, Galesic, Schulz, Ghazal, \& GarciaRetamero, 2012).

\section{Method}

Participants A total of 252 participants were recruited through Amazon Mechanical Turk (44\% female; $M_{\mathrm{AGE}}=31.4$ years, $S E M=0.62)$ and were paid U.S. $\$ .50 .{ }^{1}$ In all experiments, participation was restricted to respondents from the United States who had at least a $90 \%$ approval rating for their previous MTurk work. Participants were excluded from data analysis if they failed either of the attention check questions (described below) $(n=4)$ or gave a probability estimate of $0 \%$ or $100 \%$ $(n=2)$. In the latter case, it was assumed that participants were not seriously engaging with the problem. The final sample sizes were standard percentage $(n=44)$, standard frequency $(n=42)$, standard mixed $(n=43)$, causal percentage $(n=36)$, causal frequency $(n=43)$, and causal mixed $(n=38)$.

Design and procedure The study followed a 2 (explanation of statistics: standard vs. causal) $\times 3$ (problem format: percentage, mixed, frequency) design. Participants were randomly assigned to the six experimental conditions.

Participants were told that they would be presented with scenarios involving statistical information and would have to use this to estimate the likelihood of an event. All participants then completed one version of the mammogram problem and one version of the drug-test problem, in random order. Table 1 shows the standard and causal versions of the problem in percentage format and frequency format. The former are identical to those used by Krynski and Tenenbaum (2007, Experiment 2). Appendix B shows the mammogram problem used in the mixed format condition and all versions of the drug-test problem. In the causal conditions, a brief explanation was given of the base rate of an alternative cause (e.g., a benign cyst in the mammogram problem) and the likelihood of the data given this cause (e.g., of a positive mammogram given the cyst).

\footnotetext{
${ }^{1}$ In all experiments, the total sample to be recruited was decided in advance, and data collection terminated once this target was reached (with the small exception in Experiment 3 that replacements were obtained for two participants who did not comply with experimental instructions).
}

In the standard condition, no such explanation of false positives was given. In the percentage and mixed format conditions participants were asked to give their estimate of $p(\mathrm{H} \mid \mathrm{D})$ as an integer percentage. In the frequency format condition, the requested estimate was a frequency out of 100 . To discourage impulsive responding, participants could not enter a response for $12 \mathrm{~s}$ after the problem appeared on the screen. Participants had unlimited time to complete the problems and were encouraged to use their own calculator in deriving estimates. After completion of the judgment problems, participants were given two attention check questions involving a simple numerical judgment (e.g., "Which of the following numbers is the largest: $21,17,42,38,40$ ?") and then completed the Berlin Numeracy Test (BNT).

\section{Results and discussion}

In all of the current studies, it was of interest to discover whether the empirical evidence favored a conclusion of differences between groups in probability estimates or whether this evidence favored the null hypothesis of a lack of group differences. Hence, throughout the article we rely on Bayesian analyses of variance (BANOVAs; Rouder, Morey, Verhagen, Swagman, \& Wagenmakers, 2016) and Bayesian contingency analyses, using independent multinomial sampling (Jamil, Ly, Morey, Love, Marsman, \& Wagenmakers, 2017). Such analyses yield Bayes factors (BF) that are directly interpretable as how many times more likely one hypothesis is to have generated the observed data over another. We use the notation $\mathrm{BF}_{10}$ to refer to Bayes factors, where $\mathrm{BF}_{10}>1$ indicates support for the alternative hypothesis and $\mathrm{BF}_{10}<1$ support for the null hypothesis. For example, $\mathrm{BF}_{10}=10$ indicates the data are 10 times more likely to have been generated by the alternative hypothesis than the null hypothesis, and $\mathrm{BF}_{10}=.1$ indicates the opposite conclusion. We follow the conventions suggested by Kass and Raftery (1995) that a BF between 3 and $20(0.33$ 0.05 ) represents positive evidence for the alternative (or null) hypotheses, respectively, a BF between 21 and 150 (.049-.0067) represents strong evidence, and a BF above $150(<.0067)$ represents very strong evidence. To avoid clutter, any BF larger than 10,000 is reported as $>10,000$. All analyses were carried out using the JASP data analysis package (Love et al., 2016), using default priors.

A preliminary analysis confirmed that performance on the BNT was generally in the midrange of numerical literacy $(M=$ 2.25 out of a maximum score of $4, S E M=0.08$ ) and did not vary across experimental conditions (all $\mathrm{BF}_{10} \mathrm{~S}<0.28$ ). BNT performance was unrelated to type of categorized response given $\left(\mathrm{BF}_{10}=0.01\right)$. Most participants $(90 \%)$ did not use a calculator to solve either problem, and this did not vary across causal explanation or format conditions, (all $\mathrm{BF}_{10} \mathrm{~s}<0.57$ ). 
Open-ended estimates The normative answer for the mammogram problem was $\approx 5 \%$ (percentage) or 5 out of 100 (frequency). The normative answer for the drugtest problem was $\approx 24 \% / 24$ out of 100 (see Appendix A for the derivation). The open-ended estimates generated by participants are given in Fig. 1a-b. Because the problems had different normative solutions, we analyzed probability estimates for each separately. For convenience, these are all expressed as percentages.
Probability estimates for each problem were entered into a 2 (standard vs. causal) $\times 3$ (format) BANOVA. For the mammogram problem, it is clear from Fig. 1a that most participants did not give the correct response, with means in every condition well above the normative value. Nevertheless, there was very strong evidence of an effect of causal explanation, $\mathrm{BF}_{10}=$ $306.35, \eta_{\mathrm{p}}{ }^{2}=0.07$. Those in the causal condition generally gave lower estimates $(M=21.18 \%, S E M=2.65)$ than those in the standard condition $(M=36.36 \%, S E M=2.52)$. Because

a

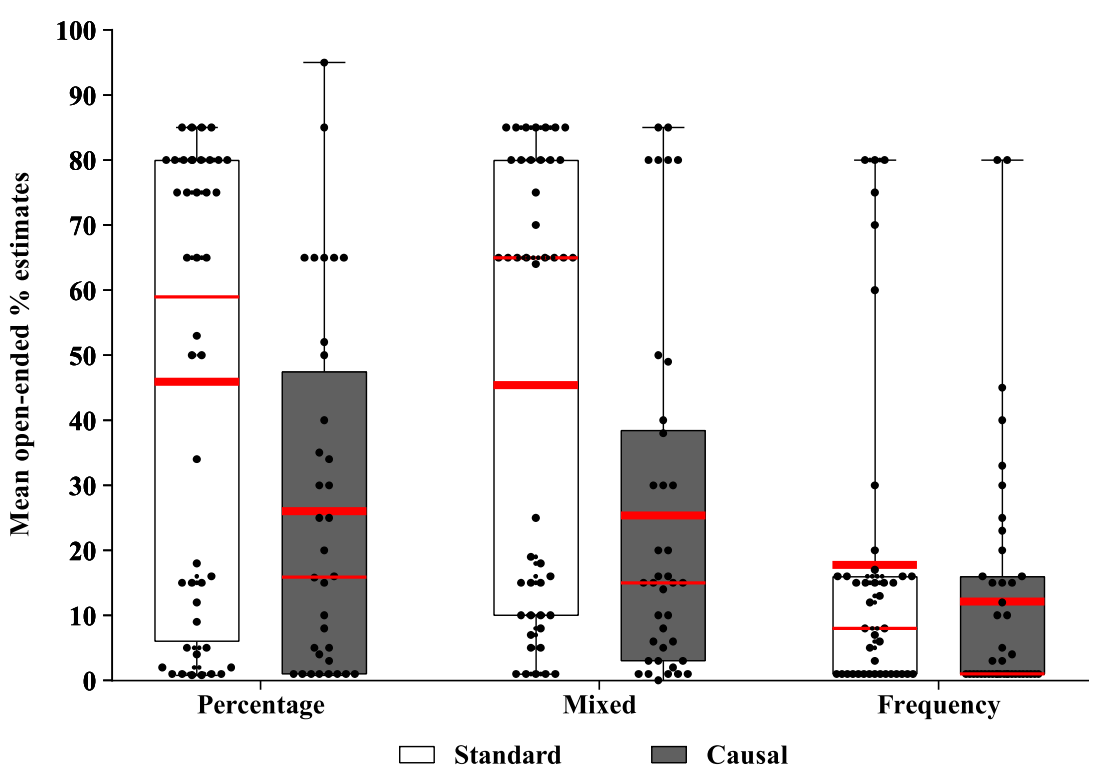

b

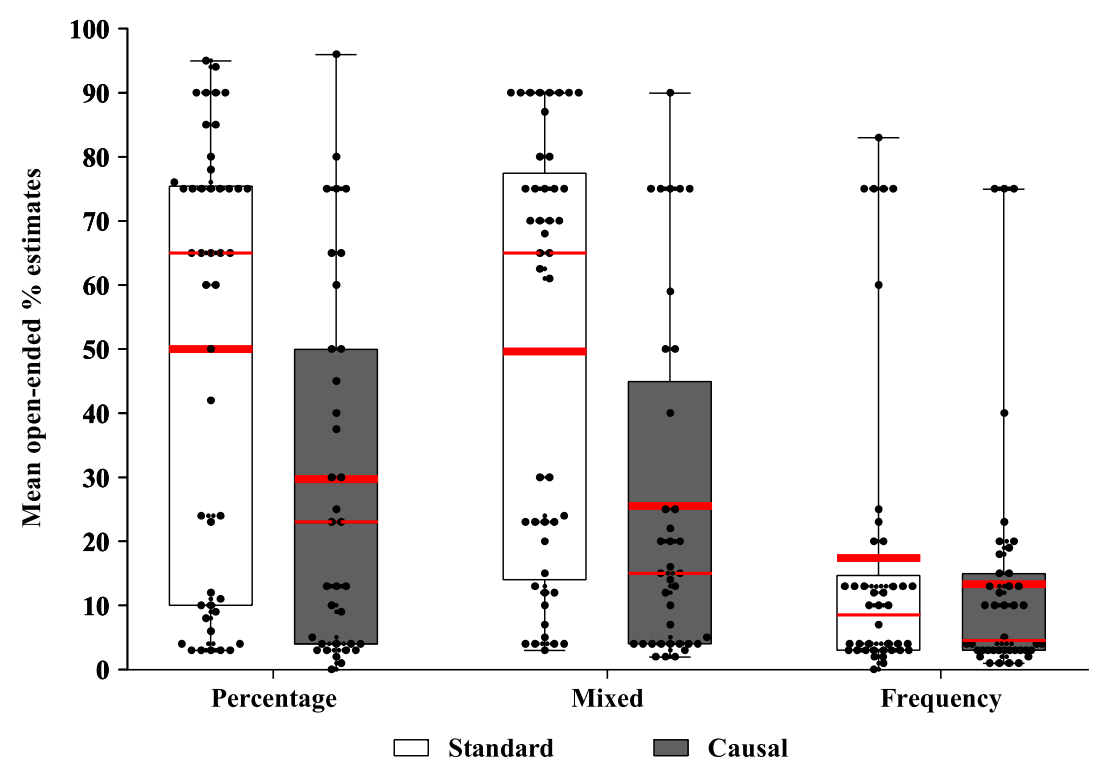

Fig. 1 a Experiment 1. Box and whisker plots of open-ended probability estimates for the mammogram problem. The thick red line within each box shows the group mean. The thin red line within each box shows the median. Individual data points are also shown. The normatively correct response was $\approx 5 \%$. b Experiment 1 . Box and whisker plots of open- ended probability estimates for the drug problem. The thick red line within each box shows the group mean. The thin red line within each box shows the median. Individual data points are also shown. The normatively correct response was $\approx 24 \%$ 
lower estimates are closer to the normative solution, this result can be seen as evidence of the weaker form of causal facilitation.

There was also very strong evidence that estimates were affected by statistical format, $\mathrm{BF}_{10}>10,000, \eta_{\mathrm{p}}^{2}=0.11$. Figure 1a shows that estimates were lower when using frequency as compared to other formats. This difference was supported by a Bayesian $t$ test between the frequency and other format conditions, $\mathrm{BF}_{10}=3.80, d=0.44$. Notably, there was indeterminate evidence for an interaction between explanation and format, $\mathrm{BF}_{10}=1.31$.

The results for the drug-test problem were generally similar (see Fig. 1b), with very strong evidence of an effect of both causal explanation, $\mathrm{BF}_{10}=1129.07, \eta_{\mathrm{p}}{ }^{2}=0.08$, and statistical format, $\mathrm{BF}_{10}>10,000, \eta_{\mathrm{p}}{ }^{2}=0.14$. In this case, there was some evidence of an interaction between causal explanation and frequency format, $\mathrm{BF}_{10}=3.51, \eta_{\mathrm{p}}{ }^{2}=0.02$. Figure $1 \mathrm{~b}$ shows that the effect of causal information was robust when averaged across the percentage and mixed format conditions, $\mathrm{BF}_{10}=1588.64, \eta_{\mathrm{p}}{ }^{2}=$ 0.08 , but not in the frequency condition, $\mathrm{BF}_{10}=0.23$. $^{2}$

Categorized estimates In order to examine the types of estimates generated, these were categorized as follows. Correct estimates were those that exactly matched the integer closest to the correct value or were within two percentage points above or below that value, making allowance for calculation errors. Underestimates were those more than two points below the correct value. Extreme overestimates were those equal to or greater than the value given by subtracting $p(\mathrm{D} \mid \neg \mathrm{H})$ from $p(\mathrm{D} \mid \mathrm{H}){ }^{3}$ Moderate overestimates were those falling between the categories of correct estimates and extreme overestimates.

Figure $2 \mathrm{a}-\mathrm{b}$ show that the proportion of correct responses was low in all conditions for both the mammogram problem (7\%-16\% of responses) and the drug-test problem (2\%-11\% of responses). For the mammogram problem, Bayesian contingency tests found evidence in favor of the null hypothesis that the proportion of normatively correct responses did not differ as a function of causal explanation, $\mathrm{BF}_{10}=0.25$, nor format, $\mathrm{BF}_{10}=0.05$. The null hypothesis was also supported in the corresponding analyses of normative responses on the drug problem (causal explanation: $\mathrm{BF}_{10}=0.14$; format: $\mathrm{BF}_{10}$ $=0.13$ ).

\footnotetext{
${ }^{2}$ It is also possible to analyze open-ended probability estimates in terms of absolute deviations from the normative response. We carried out such analyses for this and the other two experiments (see Supplementary Materials for details). These analyses did not lead to any substantive change in conclusions.

${ }^{3}$ Previous studies have used the same criteria to define extreme overestimates but referred to them as base-rate neglect errors. We avoid this terminology because there is no direct evidence that such errors are due to neglect of base rates.
}

Analyses of responses across all four categories nevertheless found that, for the mammogram problem, causal explanation had an effect on the frequency of nonnormative responses in the percentage, $\mathrm{BF}_{10}=5.43$, and mixed format conditions, $\mathrm{BF}_{10}=17.80$, but not in the frequency condition, $\mathrm{BF}_{10}=0.11$. In the percentage and mixed conditions, causal explanation was associated with lower rates of extreme errors and higher rates of moderate errors as compared with the standard explanation condition. Across both standard and causal conditions, frequency format was associated with lower rates of extreme overestimates and higher rates of underestimates and moderate errors than percentage or mixed formats were, $\mathrm{BF}_{10}=2454.0$.

The results for the drug-test problem (see Fig. 2b) were broadly similar, although, because the normative answer was higher than in the mammogram problem, the category of underestimates covered a wider range of values. Causal explanation was associated with lower rates of extreme errors and higher rates of underestimates and moderate errors in the percentage, $\mathrm{BF}_{10}=$ 8.36, and mixed format conditions, $\mathrm{BF}_{10}=8.96$, but not in the frequency condition, $\mathrm{BF}_{10}=0.01$. Across both standard and causal conditions frequency format was associated with lower rates of extreme overestimates and higher rates of underestimates, $\mathrm{BF}_{10}>10,000$.

This experiment reexamined whether providing a causal explanation of false positives improves intuitive statistical judgments. Our results show that it depends on how performance is measured. Like McNair and Feeney $(2014,2015)$, we failed to replicate the large effects of causal explanation on normative responding reported in Krynski and Tenenbaum (2007, Experiment 2 ). On the mammogram problem, for example, we found only $11.1 \%$ of those in the causal percentage format condition gave a normatively correct estimate as compared with over $40 \%$ of Krynski and Tenenbaum's participants. However, causal explanation did reduce the magnitude of overestimation errors, consistent with the weak causal facilitation hypothesis. In the causal condition, the modal response was nonnormative but was less likely to be an extreme overestimate than in the standard condition. These results held true for both mammogram and drug test problems.

In this study, we employed the standard and causal versions of the mammogram problem used by Krynski and Tenenbaum (2007, Experiment 2). In this case, only the causal condition contained explicit statements of both the base rate of women with a cyst, $p(\ulcorner\mathrm{H})$, and the likelihood of a positive mammogram given a cyst, $p(\mathrm{D} \mid-\mathrm{H})$. To calculate the false positive rate, participants in the causal condition had to multiply these statistics. Hence, deriving the normative answer involved an extra computational step in the causal condition as compared 

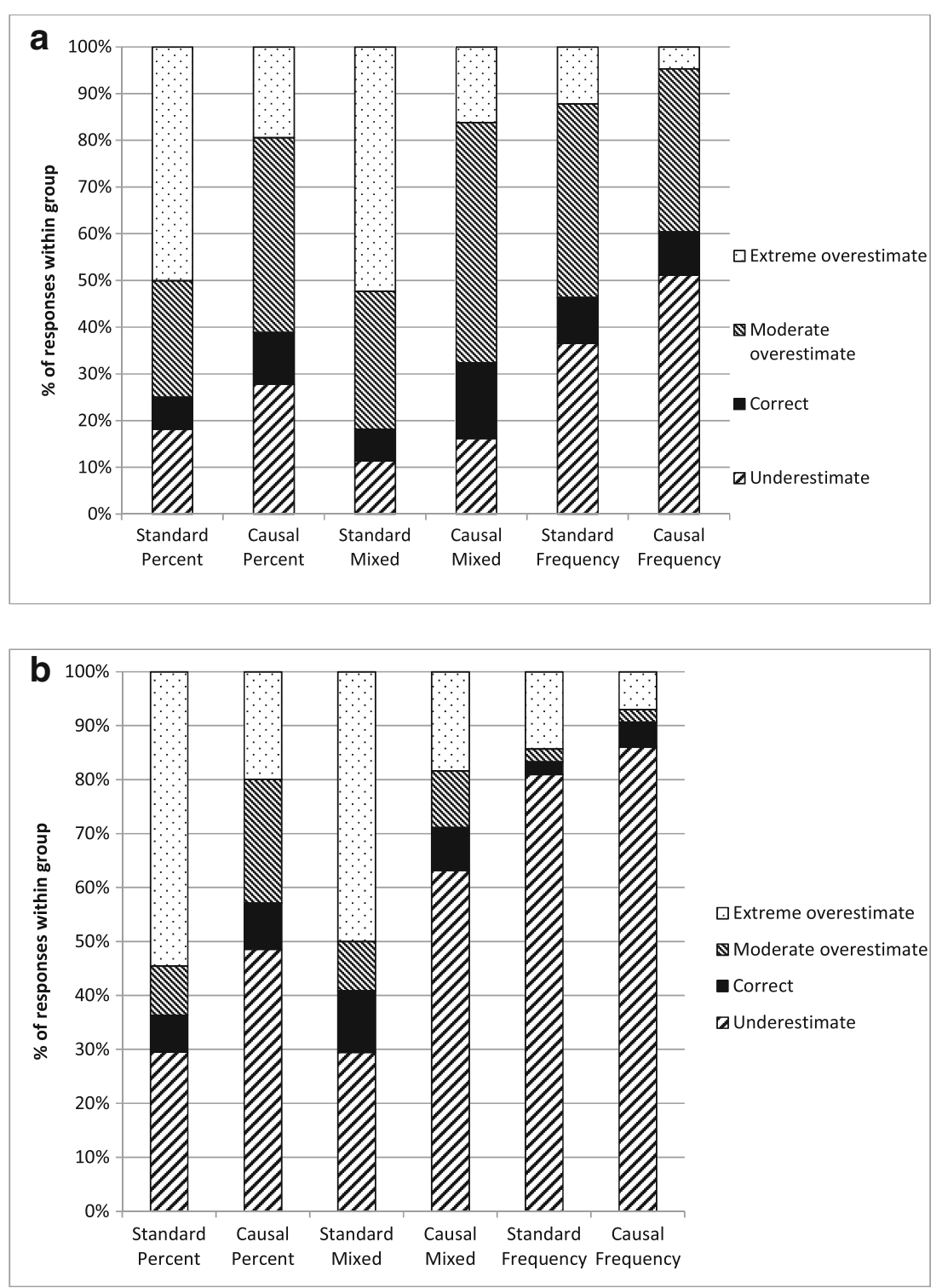

Fig. 2 a Experiment 1. Mammogram problem. Percentage of estimates in each response category in each condition. Estimate ranges: underestimate $<3 \%$; correct 3\%-7\%; moderate overestimate $8 \%-64 \%$; extreme overestimate $\geq 65 \%$. b Experiment 1 . Drug-test problem.

with the standard. This may have worked against causal facilitation of normative responding. This problem was addressed in Experiment 2 where we compared performance in the standard mammogram with that in two different causal conditions: (a) a replication of the current four-parameter causal condition and (b) a simplified three-parameter version causal condition, which included only a single false positive statistic.

Experiment 1 also examined whether weak causal facilitation persisted when statistical information was presented in frequency format. Mixed results were found on this issue. For open-ended estimates in the mammogram problem, the effects of causal explanation and frequency format were additive. In the drug-test
Percentage of estimates in each response category in each condition. Estimate ranges: underestimate $<22 \%$; correct $22 \%-26 \%$; moderate overestimate $27 \%-64 \%$; extreme overestimate $\geq 65 \%$

problem, however, the effects of causal explanation were limited to the percentage format and mixed conditions. This issue was reexamined in Experiment 2 where, as explained below, the effects of causal explanation were tested using percentage format and an enhanced frequency format.

The lack of facilitation of performance in the mixed condition confirms our suggestion that McNair and Feeney's (2014) null results relating to frequency formats were the result of using different formats for expressing problem statistics and generating solutions (cf. Cosmides \& Tooby, 1996). In contrast, presenting statistics and solutions as frequencies did reduce the size of numerical probability estimates but did not increase 
the rate of normative responding. Figure $2 \mathrm{a}-\mathrm{b}$ shows that this was largely because use of frequency format led to a shift from overestimation of the conditional probability to underestimation. Participants in the frequency format condition often just reported the base rate as their answer ( $42 \%$ of participants in the frequency condition).

The failure to find an increase in normative responding is somewhat surprising, given previous findings (e.g., Cosmides \& Tooby, 1996; Evans et al., 2000), and may have been due to the use of a less than optimal presentation of the relevant frequencies. A number of researchers (e.g., Barbey \& Sloman, 2007; Fiedler, Brinkmann, Betsch, \& Wild, 2000) have suggested that facilitation of performance in Bayesian problems is most likely to be achieved when (a) frequencybased statistics are presented with reference classes that indicate the nested structure of the various groups described in the problem, and (b) frequencies simplify problem computation by eliminating the need to use base rates. The use of a common reference class of 100 in the current frequency problems did not meet these criteria. This issue was addressed in Experiment 2 , where we employed a modified frequency format.

\section{Experiment 2}

This study reexamined the impact of causal explanation of false positives on performance in Bayesian problems. Focusing on the mammogram problem, we again presented participants with standard or causal explanation conditions like those in Experiment 1. In this case, however, we added a three-parameter causal condition, where false positives were explained as being caused by a benign cyst, but only a single false positive rate $(15 \%)$ was given. Hence, this threeparameter causal condition was equivalent to the standard condition in terms of computational complexity (see Table 2 for details).

It may be that this simplified version of the causal condition leads to greater facilitation of performance over the standard than our original four-parameter causal preparation. However, it is also possible that this change in presentation of causal information could work in the opposite direction. If the weak-facilitation effect observed in Experiment 1 was largely due to increased attention to the two statistics associated with the causal explanation, then the use of a simplified causal story and a single statistic may produce less facilitation, despite the reduction in computational complexity.

McNair and Feeney (2014) found that neither a threeparameter nor a four-parameter causal version of the mammogram problem led to increased levels of
Table 2 New standard and causal judgment problems in percentage format (upper panel) and frequency format (lower panel) used in Experiment 2 (instructions in italics differed between the standard and causal versions)

Mammogram problem (causal three-parameter, percentage format)

The following statistics are known about women at age 60 who participate in a routine mammogram screening for breast cancer:

$1 \%$ of the women have breast cancer at the time of screening.

Of those with breast cancer, $80 \%$ receive a positive result on the mammogram.

Of women without breast cancer, $15 \%$ will receive a positive result on the mammogram because they had a benign cyst at the time of screening.

Suppose a woman gets a positive result during a routine mammogram screening. Without knowing any other symptoms, what is the probability that she has breast cancer?

\section{Mammogram problem (modified frequency format)}

The following statistics are known about women at age 60 who participate in a routine mammogram screening for breast cancer:

10 in every 1000 women have breast cancer at the time of screening.

8 in every 1,000 women have breast cancer and will receive a positive result on the mammogram.

150 in every 990 women without breast cancer will receive a positive result on the mammogram. [Standard]

150 in every 990 women without breast cancer have a benign cyst at the time of screening and will receive a positive result on the mammogram. [Causal-3 PARAMETER]

300 in every 990 women have a benign cyst at the time of screening. Of those with a benign cyst, half will receive a positive result on the mammogram. [Causal-4 PARAMETER]

Imagine that we have selected a random sample of 100 women over 60 . Without knowing any other symptoms, on average how many women who receive a positive result during a routine mammogram screening do you expect to have breast cancer.

normative responding relative to a standard condition. However, they did not compare the magnitude of solution estimates and so could not detect the type of weak facilitation effect we observed in Experiment 1. The current experiment therefore offered an opportunity to reexamine the impact of the different levels of causal explanation on performance in the mammogram problem.

In this study, we also modified the frequency format condition with a view to highlighting nested set relations (cf. Barbey \& Sloman, 2007; Lesage et al., 2013) and simplifying the computation of the normative solution (cf. Fiedler et al., 2000). The modified frequency format is illustrated in Table 2. Note that the use of the reference class of 1,000 or 990 women highlights the differences between the relative frequencies of those with breast cancer who receive a positive test and those without cancer/with a cyst who receive a positive test. Moreover, in this version, the solution can be computed from the given statistics without reference to the base rate, that is, $p(\mathrm{H} \mid \mathrm{D})=8 /(8+150)$. 


\section{Method}

Participants A total of 360 participants were recruited through Amazon Mechanical Turk $\left(41 \%\right.$ female; $M_{\mathrm{AGE}}=$ 37.7 years, $S E M=1.41$ ) and were paid U.S. $\$ .50$. Participants were excluded from data analysis if they failed either of the attention check questions $(n=9)$, reported that they had previously completed a similar task $(n=31)$, or gave a probability estimate of $0 \%$ or $100 \%(n=4)$. The final sample sizes were standard percentage $(n=55)$, standard frequency $(n$ $=49)$, causal three-parameter percentage $(n=50)$, causal three-parameter frequency $(n=59)$, causal four-parameter percentage $(n=53)$, and causal four-parameter frequency ( $n$ $=50)$.

Design and procedure The study followed a 3 (explanation of statistics: standard, causal three-parameter, causal four-parameter) $\times 2$ (format: percentage, frequency) design . Participants were randomly assigned to the experimental conditions.

The procedure was similar to that used for the mammogram problem in Experiment 1, except for the following changes. As well as the causal condition used in the earlier study (hereafter the causal four-parameter condition), we developed a three-parameter version of the causal mammogram script (see Table 2). Like the standard version, this script provided only a single statistic for the false positive rate $(15 \%)$ but provided a causal explanation for the statistic. The scripts for the frequency conditions used in this study were revised to convey the nested nature of the various components of the mammogram problem (see Table 2). In the current frequency conditions, a small adjustment was made to the denominators for $p(\ulcorner\mathrm{H})$ and $p(\mathrm{D} \mid\ulcorner\mathrm{H})$, to take account of frequency of women with cancer (i.e., denominators $=1,000-10=990$ ).

\section{Results and discussion}

A preliminary analysis confirmed that performance on the BNT was generally in the midrange of numerical literacy $(M$ $=2.46$ out of a maximum score of $4, S E M=0.07$ ) and did not vary across experimental conditions $\left(\mathrm{BF}_{10} \mathrm{~s}<0.31\right)$. Bayesian contingency analysis found that BNT performance was unrelated to the type of categorized response given, $\mathrm{BF}_{10}=0.11$. However, there was some suggestion in the data of an asymmetric relationship between high numeracy and normative performance. Only a relatively small proportion of those who received the maximum score on the BNT gave the normatively correct response (23\%). However, of those who gave the correct response, a substantial proportion received the maximum BNT score (78\%). In other words, high levels of numerical ability increased the likelihood of normative responding, but this was not sufficient to ensure such responding.

Nevertheless, the largest proportion of those who gave the normatively correct response had a maximum BNT score of 4 (23.4\% of all those with correct responses as compared with 7\%-13\% for those with BNT scores between 1 and 3). Most participants $(n=253,80.1 \%)$ did not use a calculator to solve the problem, and this did not vary across causal explanation or format conditions, $\mathrm{BF}_{10} \mathrm{~s}<0.28$. Calculator use, however, did improve performance, $\mathrm{BF}_{10}=4,312.0$. Thirty-three percent of those who used a calculator gave the correct response as compared with $9.1 \%$ of those who did not.

\section{Open-ended estimates}

The mean estimates for each condition are given in Fig. 3, expressed as percentages. These were entered into a 3 (explanation) $\times 2$ (format) BANOVA. As is clear from Fig. 3, there was very strong evidence of an effect of format, $\mathrm{BF}_{10}>10,000, \eta_{\mathrm{p}}{ }^{2}=0.27$. Those given the revised frequency format version of the problem gave lower estimates than those who received the standard version. Overall, there was only weak evidence of an effect of causal explanation on probability estimates, $\mathrm{BF}_{10}=2.4$, $\eta_{\mathrm{p}}{ }^{2}=0.02$. Nevertheless, there was evidence that estimates given in the causal four-parameter percentage condition were lower than those in the standard condition, $\mathrm{BF}_{10}=$ 3.64, $\eta_{\mathrm{p}}^{2}=0.06$. Notably, no evidence was found of differences between the estimates given in the causal three-parameter and standard conditions, $\mathrm{BF}_{10}=0.16$.

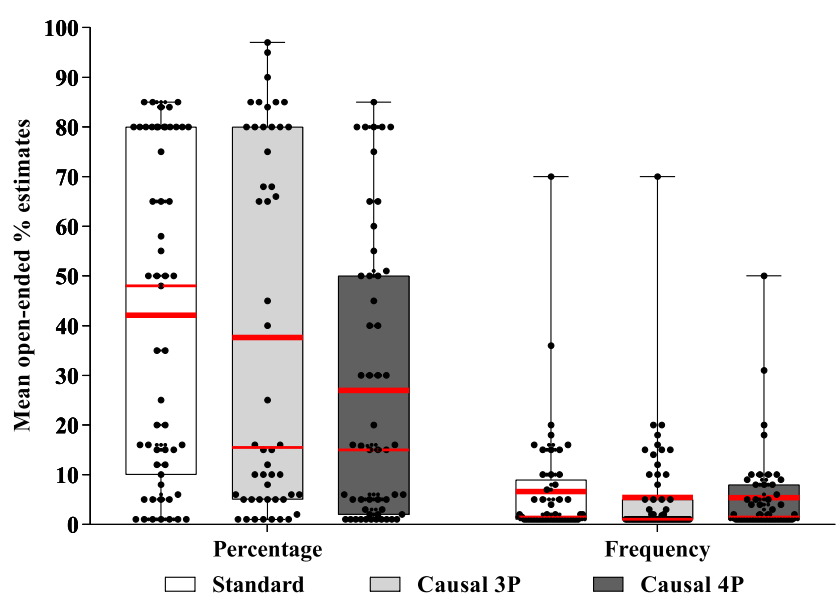

Fig. 3 Experiment 2. Box and whisker plots of open-ended probability estimates. The thick red line within each box shows the group mean. The thin red line within each box shows the median. Individual data points are also shown. The normatively correct response was $\approx 5 \% .3 \mathrm{P}=$ three parameter version; $4 \mathrm{P}=4$ parameter version 
Categorized estimates Open-ended estimates were categorized in the same way as Experiment 1 (see Fig. 4). We again used Bayesian contingency tests to compare the frequency of normative responses between conditions. These found evidence in favor of null effects of causal explanation, $\mathrm{BF}_{10}=$ 0.03 , and frequency format, $\mathrm{BF}_{10}=0.12$, on normative responding.

Nevertheless, analysis of responses across the four response categories found that statistical format had a large effect on the types of nonnormative solutions that were generated, $\mathrm{BF}_{10}>10,000$. Figure 4 shows that those given the mammogram problem in a frequency format were more likely to underestimate the problem solution and were less likely to give an extreme overestimate than those given the percentage format. Within the percentage format conditions, the type of problem solution differed depending on whether people were given the standard description of false positives or were given the fourparameter causal explanation of false positives. Bayesian contingency analysis found some evidence of a difference in response patterns between the standard and causal fourparameter condition, $\mathrm{BF}_{10}=2.47$. However, the evidence indicated there was no difference between the response patterns of the standard and causal three-parameter condition, $\mathrm{BF}_{10}=0.05$. Within the frequency format conditions, the evidence indicated that there were no differences between the types of responses given in the standard and either of the causal conditions, $\mathrm{BF}_{10}=0.02$ and $\mathrm{BF}_{10}=0.01$.

The results for the standard and causal four-parameter, percentage format condition replicated the weak causal facilitation effect found in the corresponding conditions of Experiment 1. This type of causal explanation led to less extreme overestimates and more underestimates of the normative solution than was the case in the standard condition but produced no change in levels of normative responding. In contrast, no reliable differences in responding were found between the three-parameter version of causal explanation and the standard explanation. This null effect may seem puzzling given that computing the normative response involved fewer steps in the threeparameter version than in the four-parameter version. However, this pattern is consistent with an interpretation of the weak facilitation effect as at least partly driven by increased attention to the false positive statistics. In this scenario, people may simply anchor their estimates on one or both of the corresponding values, with little attempt to integrate these with the other relevant statistics. Experiment 3 follows up on this possibility by using process-tracing methods to uncover the specific reasoning behind estimates generated by those in the standard and causal four-parameter conditions.

The modified frequency condition led to a substantial downward shift in probability estimates but no increase in normative accuracy. In this frequency condition, those in the standard and causal conditions gave similar patterns of estimates, with a very high proportion of underestimates (62\% of all responses in the frequency groups). In most cases, people simply reported the base rate of 1 in 100 (54\% of all responses in the frequency groups). Similar increases in base rate overuse when frequency formats are used have been reported by Evans et al. (2000, Experiment 1) and Sloman, Over, Slovak and Stibel (2003). It appears that expressing statistics in a frequency format can lead participants to substitute one kind of biased reasoning - focusing primarily on $p(\mathrm{D} \mid \mathrm{H})$ - with another (focusing on the base rate). Sloman et al. (2003) suggest that the use of a common reference class in frequency format problems is only an indirect cue to the relevant set relations between subgroups and hence may often be misunderstood. Other methods (e.g., diagrammatic representation of problem

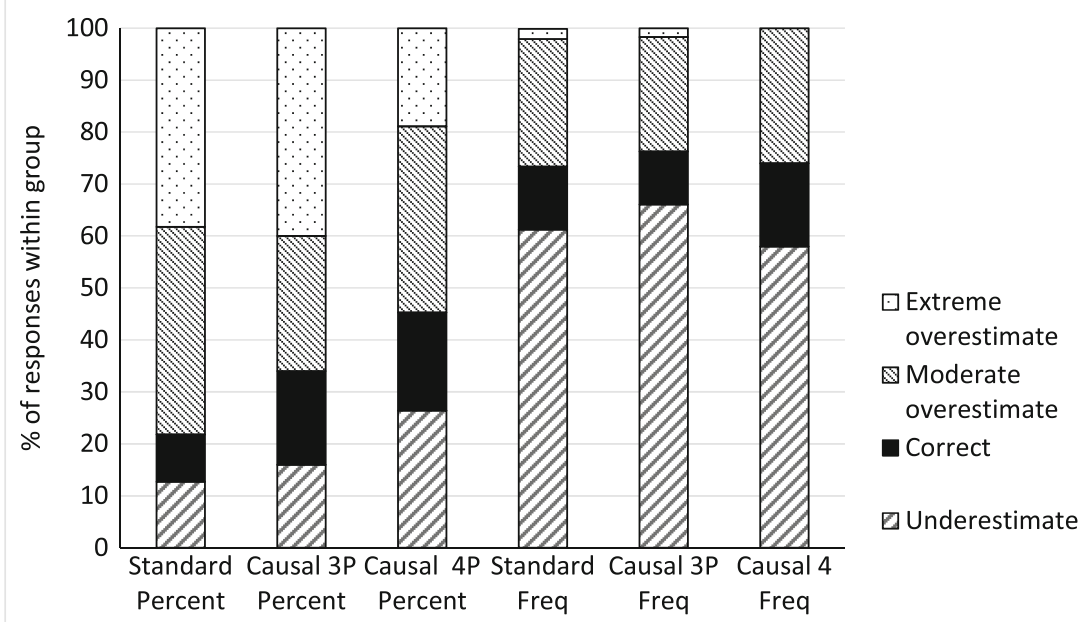

Fig. 4 Experiment 2. Percentage of estimates in each response category. $3 \mathrm{P}=$ three parameter version; $4 \mathrm{P}=$ four parameter version. Estimate ranges: underestimate $<3 \%$; correct $3 \%-7 \%$; moderate overestimate $8 \%-64 \%$; extreme overestimate $\geq 65 \%$ 
components) may offer a more direct route to improved performance on Bayesian reasoning problems.

\section{Experiment 3}

Experiments 1 and 2 found that explaining the causal origin of $p(\ulcorner\mathrm{H})$ and $p(\mathrm{D} \mid\ulcorner\mathrm{H})$ does not increase normative statistical estimation but does reduce the magnitude of estimation errors. This could reflect a weaker version of Krynski and Tenenbaum's (2007) causal Bayesian process. The additional causal information could lead people to complete more steps in computing the Bayesian solution than they would otherwise, even though they do not reach the exact solution. Alternately, the reduction in error might reflect a heuristic process, such as increased attention to the false positive statistics during the problem solution. A mixture of such strategies across different individuals is also possible.

These alternative accounts cannot be differentiated by examination of numerical estimates alone. Individual estimates may be the end product of a number of different reasoning strategies. For example, in the earlier studies an open-ended response of " $15 \%$ " in the mammogram problem may have been an approximate estimate of the denominator of Bayes rule-that is, $(0.01 \times 0.8)+(0.99 \times 0.15)=0.1565$. Alternately, it may have simply been a repetition of the given false positive rate. Moreover, the reasoning behind many of the estimates generated in Experiments 1 and 2 remains opaque. For example, several participants in Experiment 1 $(n=9)$ gave an estimate of " $10 \%$ " in the mammogram problem, but it is unclear how they arrived at this figure.

Experiment 3 therefore aimed to provide a clearer specification of the reasoning strategies used in the standard and causal four-parameter versions of the mammogram task. This was achieved using a process-tracing approach in which participants gave verbal reports of their problem-solving strategies while estimating $p(\mathrm{H} \mid \mathrm{D})$. A comparison of problemsolving strategies in the standard and causal conditions should reveal the extent to which differences in probability estimates reflect the adoption of Bayesian strategies, non-Bayesian heuristic strategies, or a mixture of strategies.

Process-tracing methods have proved useful in uncovering individual differences in reasoning strategies on tasks involving multi-attribute choice (e.g., Bröder, 2003; Levin \& Jasper, 1995) and risky decision making (Cokely \& Kelly, 2009). To our knowledge, the current study represents the first attempt to use process tracing to study the effects of causal explanation on Bayesian reasoning. Process tracing typically involves one-on-one interaction between experimenters and participants. Hence, this experiment was carried out with an inhouse laboratory sample (university undergraduates) rather than with online participants. This meant that participants were generally younger and more educationally homogeneous than in the earlier studies.

\section{Method}

Participants Eighty psychology undergraduates (45 females, $M_{\mathrm{AGE}}=19.85$ years, $S D=3.74$; range: $17-41$ years) participated for course credit and were randomly allocated to the standard or causal condition. One was excluded because they gave a probability estimate of $100 \%$ but failed to justify this answer. Another was excluded because they failed to follow the protocol instructions. These participants were replaced.

Procedure Participants were tested individually in a quiet room. They were presented with the standard or causal percentage format versions of the mammogram problem from Experiment 1 (i.e., the causal four-parameter version). ${ }^{4}$ The think-aloud protocol was explained before participants read the problem descriptions. Protocol instructions emphasized that participants should "say whatever you are thinking as you try to solve the problem" and to report "as much as possible about what you are thinking." A short video clip was played that showed a person giving a detailed think-aloud protocol while trying to solve a moderately difficult anagram. The participant was then given an opportunity to practice thinking aloud while answering two tasks. The first task was a different anagram and the second task was a multiplication question. During this practice, the experimenter provided feedback encouraging participants to verbalize.

At the commencement of the main task, participants were told to read the problem aloud and to verbalize any thoughts they had while reading. They were then asked to verbalize their thoughts while solving the problem (see Appendix $\mathrm{C}$ for detailed instructions). A calculator and pen-and-paper were provided, but participants were instructed to verbalize all calculation steps when using these aids. An experimenter was present during the entire test session and provided general prompts throughout if the participants stopped verbalizing for more than 10 seconds. At the end of the task participants had to provide an estimate of $p(\mathrm{H} \mid \mathrm{D})$ as a percentage and rate their confidence $(1=$ not at all confident, $10=$ very confident $)$ that their answer was correct. Participants also completed a computer-based version of the BNT. The session was recorded using a Zoom ${ }^{\odot}$ H1 Handy audio recorder.

Scoring As in the previous study, numerical estimates of $p(\mathrm{H} \mid \mathrm{D})$ were compared between standard and causal groups. Numerical estimates were again assigned to one of four response categories

\footnotetext{
${ }^{4}$ During testing, some participants in the causal condition were uncertain about the meaning of "benign cyst." Hence, the instructions were adjusted to refer to a "harmless cyst/growth." This had little impact on performance within the causal group, with no difference in mean estimates given by those receiving the original or modified instructions, $\mathrm{BF}_{10}=0.4$.
} 
(correct, underestimate, moderate overestimate, extreme overestimate).

Verbal process-tracing protocols were transcribed and coded as follows: During the problem solution, we noted whether participants used pen and paper and/or a calculator during their problem solution. We also noted whether participants predominantly used percentages, proportions, or frequencies in their numerical calculations, and whether they spontaneously generated tree or set diagrams during the solution process.

Individual protocols were scored in two different ways. First, solutions were tabulated according to which components of Bayes' theorem, or combination of components, were mentioned in verbatim reports or written notes. To permit cross-group comparisons, in the causal group, mention of either of the statistics corresponding to $p(-\mathrm{H})$ or $p(\mathrm{D} \mid-\mathrm{H})$ (or both), were counted once as a mention of the false positive rate. This was necessary because, like Krynski and Tenenbaum (2007, Experiment 2), two statistics relevant to the false positive rate were described in the causal condition (see Table 1).

Second, we tabulated specific solution strategies that could be identified from the protocols and were used by at least $5 \%$ of the participants. Seven discrete solution strategies were identified: (1)-(3) repeating one of the statistical figures given in the problem (base rate, likelihood, false positive rate); (4) subtracting the false positive rate from the likelihood (i.e., $80-15=65$ or 80 $-(30 \times 0.5)=65)$; (5) multiplying the likelihood and false positive rates $(0.8 \times 0.15)$ or $0.8 \times(0.3 \times 0.5)=$ .12); (6) calculation of the Bayesian numerator (i.e., $0.01 \times 0.8)$ or denominator (i.e., $(0.01 \times 0.8)+(0.99$ $\times 0.15)$ ), but not both; and (7) normative Bayesian solution. ${ }^{5}$ Strategies that did not fall into these categories were designated other.

An independent rater scored 20 protocols (10 randomly selected from each of the standard and causal conditions) to check the reliability of the protocol coding system. Interrater agreement was generally high (agreement ranged from $79 \%$ to $100 \%$ for individual response categories). Disputes over coding were resolved via discussion between raters.

\section{Results and discussion}

The standard $\left(M_{\mathrm{BNT}}=2.73, S E M=0.18\right)$ and causal groups $\left(M_{\mathrm{BNT}}=2.70, S E M=0.18\right)$ did not differ in numeracy, $\mathrm{BF}_{10}=$ 0.23 . BNT scores in this study however tended to be higher than those of participants in Experiments 1 and 2, which

\footnotetext{
${ }^{5}$ For the last two strategies, we included solutions that did not multiply the false-positive rate by $0.99, p(\neg \mathrm{H})$, in the denominator, since this step made little difference to the final estimate.
}

presumably reflects the use of a highly educated university sample. The causal and standard groups did not differ in confidence in the accuracy of their probability estimates (standard: $M=4.83$; causal: $M=4.7$ ), nor in the time taken to derive an answer to the mammogram problem (standard: $M$ $=363 \mathrm{~s}$; causal: $M=408 \mathrm{~s}$ ), $\mathrm{BF}_{10}=0.24$ and 0.31 , respectively. Most participants used a pen and paper while answering the problem (standard: 95\%, causal: 90\%). Notably, the proportion of participants who used a calculator $(n=38,47.5 \%$ overall) was higher than in previous experiments (recall that previously, personal calculator use was encouraged, but unlike Experiment 3, participants were not provided with a calculator). Moreover, calculator use was more common in the standard condition ( $n=23,57.5 \%$ of participants) than in the causal ( $n=15,37.5 \%$ of participants). For this reason, calculator use was included as a factor in subsequent analyses. Those who used a calculator were no more numerate (calculator used: $M_{\mathrm{BNT}}=2.76$; not used: $M_{\mathrm{BNT}}=2.67$ ) or confident in their probability estimates (calculator used: $M=4.81$; not used: $M=4.71$ ) than those who did not, $\mathrm{BF}_{10}=0.24$ and 0.25 , respectively. However, they did take longer in deriving a solution (calculator used: $M=479 \mathrm{~s}$; calculator not used: $M=$ $302 \mathrm{~s}), \mathrm{BF}_{10}=24.46$.

Open-ended estimates Probability estimates were entered in a 2 (condition: standard vs. causal) $\times 2$ (calculator used: yes or no) BANOVA. The analysis found weak evidence in favor of a difference in estimates between conditions, $\mathrm{BF}_{10}=2.87, \eta_{\mathrm{p}}{ }^{2}$ $=0.07$. The causal group generally gave lower estimates $(M=$ $21.4 \%, S E M=4.40)$ than those in the standard group $(M=$ $36.98 \%, S E M=4.30$ ). This replicates the weak causal facilitation effects found in the first two experiments. Those who used a calculator generally gave lower estimates $(M=19.0 \%$, $S E M=4.55)$ than those who did not $(M=39.3 \%, S E M=$ 4.30), $\mathrm{BF}_{10}=11.18, \eta_{\mathrm{p}}{ }^{2}=0.11$, but there was indeterminate evidence of an interaction between condition and calculator use, $\mathrm{BF}_{10}=1.30$.

Categorized estimates Open-ended responses were classified in the same way as previous studies, except that responses that fell in the correct range were checked against participant solution protocols to ensure that these responses were the product of Bayesian computation. Hence, assignment to the correct category was based on more stringent criteria in this study than in the earlier experiments. Two participants who generated an open-ended response of "5" were not classified as correct because their protocols showed that they arrived at the result in a non-Bayesian way (in both cases, the solution given was $p(\mathrm{H} \mid \mathrm{D})=100-80-15=5$ ). These participants were excluded from this general analysis of response categories but were included in the more fine-grained protocol analysis below, where their solutions were classified as other. 
Categorized estimates are shown in Fig. 5. Because of the large effect of calculator use on performance, subsequent comparisons between the types of answers given in the causal and standard groups were separated according to calculator use. It is notable that only those who used a calculator gave a normative response (standard: $n=5$, causal, $n=2$ ). A Bayesian contingency test confirmed that this was evidence of a difference in normative responding based on calculator use, $\mathrm{BF}_{10}=12.16$. However, as in previous studies, the evidence favored a null effect of causal explanation on the frequency of normative responses, $\mathrm{BF}_{10}=0.30$.

As in previous studies, we examined whether the pattern of categorized responses differed between the causal and standard groups. A Bayesian contingency test found evidence that participants in the causal and standard groups who did not use a calculator had different response patterns, $\mathrm{BF}_{10}=11.13$. Figure 5 shows that those in the causal condition were more likely than those in the standard to give a moderate overestimate or underestimate, and less likely to give a large overestimate. When a calculator was used, there was no clear evidence of a difference in causal and standard response patterns, $\mathrm{BF}_{10}=0.94$. The group patterns found for those who did not use a calculator are broadly similar to those found in the percentage format conditions in Experiments 1 and 2.

As in the previous studies, BNT performance was unrelated to type of categorized response given, $\mathrm{BF}_{10}=$ 0.09. There was no evidence or there was only indeterminate evidence of a relationship between BNT and types of responses, when participants were segregated according to calculator use, $\mathrm{BF}_{10}$ 's $<1.1$. Nevertheless, as in Experiment 2, there appeared to be an asymmetric relation between numerical performance and normative responding. Only a relatively small proportion of those who received the maximum score on the BNT gave the normatively correct response $(23 \%)$. However, of those who gave the correct response, a substantial proportion received the maximum BNT score (78\%). A high level of numeracy again appeared to be a necessary but not sufficient condition for normative performance on the mammogram task.

In most respects, these results replicate the weak facilitation pattern found in Experiments 1 and 2. When the causal origin of false positives was explained, people gave lower conditional probability estimates overall than in the standard condition and gave fewer extreme overestimates. However, the additional causal information did not increase the rate of normative solutions.

Process-tracing protocol analysis During the problem solution, the groups did not differ in their generation of tree or set diagrams (standard: $25 \%$ of participants, causal: $20 \%$ of participants), or in translation of the problem into a frequency format (standard: $25 \%$ of participants, causal: $22.5 \%$ of participants).

Table 3 shows the components of Bayes' rule, or combinations of components, that were mentioned in participant protocols, segregated by calculator use. When a calculator was used, there was indeterminate evidence of a difference between the standard and causal groups in use of Bayesian components, $\mathrm{BF}_{10}=1.02$. When no calculator was used, there was strong evidence of a difference between the types of the Bayesian components mentioned during solution by standard and causal participants, $\mathrm{BF}_{10}=21.72$. We need to be cautious in interpreting this result because of the small cell sizes. Inspecting the table, however suggests at least one distinct group difference in the Bayesian components mentioned by those who did not use a calculator. Those in the causal group were more likely to generate solutions that focused exclusively on the false positive rate (eight out of 25 participants) whereas those in the standard group never generated such solutions.

A more detailed picture of the Bayesian and non-Bayesian solution strategies employed in each group is given in Table 4. This table shows how participants used or combined the various statistics given in the problem to reach a solution.

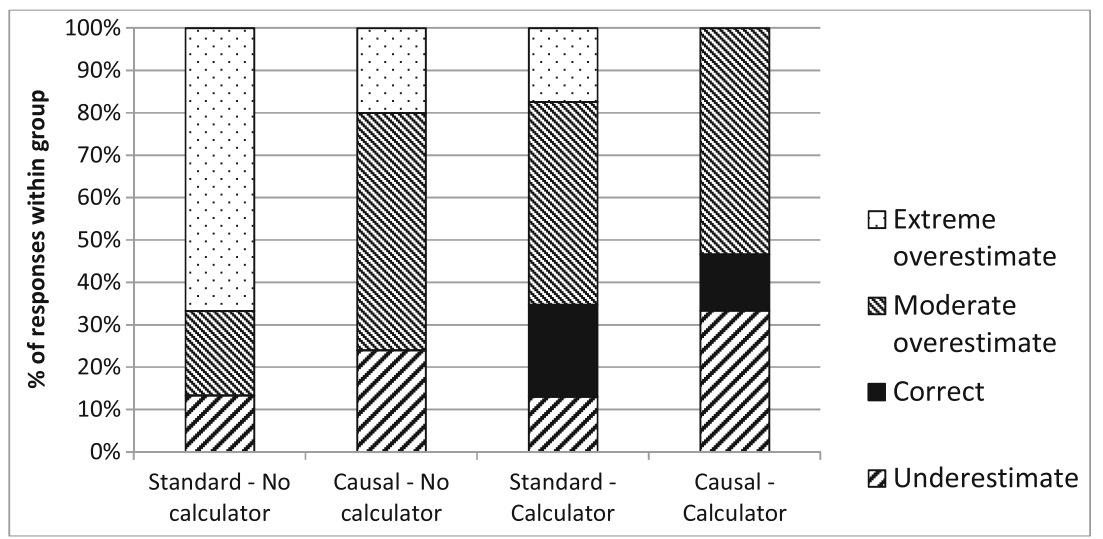

Fig. 5 Experiment 3. Percentage of estimates in each response category. Estimate ranges: underestimate $<3 \%$; correct 3\%-7\%; moderate overestimate $8 \%-64 \%$ : extreme overestimate $\geq 65 \%$. 
Table 3 Experiment 3. Frequency (and within-group proportion) of Bayesian components mentioned in problem solution

\begin{tabular}{|c|c|c|c|c|c|c|c|}
\hline Condition & $\begin{array}{l}\text { Target base } \\
\text { rate } p(\mathrm{H})\end{array}$ & $\begin{array}{l}\text { Likelihood of } \\
\text { target } p(\mathrm{D} \mid \mathrm{H})\end{array}$ & $\begin{array}{l}\text { False positive rate } \\
p(\mathrm{D} \mid \neg \mathrm{H}) \text { and/or } p(\neg \mathrm{H})\end{array}$ & $\begin{array}{l}p(\mathrm{H}) \text { and } \\
p(\mathrm{D} \mid \mathrm{H})\end{array}$ & $\begin{array}{l}p(\mathrm{D} \mid \mathrm{H}) \text { and } \\
p(\mathrm{D} \mid \neg \mathrm{H})\end{array}$ & $\begin{array}{l}p(\mathrm{H}) \text { and } p(\mathrm{D} \mid \mathrm{H}) \\
\text { and } p(\mathrm{D} \mid \neg \mathrm{H})\end{array}$ & Other \\
\hline $\begin{array}{l}\text { Standard/No } \\
\quad \text { calculator }(n=17)\end{array}$ & $2(11.8 \%)$ & $6(35.3 \%)$ & 0 & 0 & $5(29.4 \%)$ & $2(11.8 \%)$ & $2(11.8 \%)$ \\
\hline $\begin{array}{l}\text { Causal/No calculator } \\
\quad(n=25)\end{array}$ & $4(16.0 \%)$ & $3(12.0 \%)$ & $8(32.0 \%)$ & $3(12.0 \%)$ & $3(12.0 \%)$ & $4(16.0 \%)$ & 0 \\
\hline $\begin{array}{l}\text { Standard/Calculator } \\
\quad(n=23)\end{array}$ & 0 & 0 & $1(4.3 \%)$ & $1(4.3 \%)$ & $5(21.7 \%)$ & $15(65.2 \%)$ & $1(4.3 \%)$ \\
\hline $\begin{array}{l}\text { Causal/Calculator } \\
\quad(n=15)\end{array}$ & 0 & 0 & $1(6.7 \%)$ & $3(20.0 \%)$ & $4(26.7 \%)$ & $5(33.3 \%)$ & $2(13.3 \%)$ \\
\hline Total & $6(7.5 \%)$ & $9(11.3 \%)$ & $10(12.5 \%)$ & $7(8.8 \%)$ & $17(21.3 \%)$ & $26(32.5 \%)$ & $5(6.3 \%)$ \\
\hline
\end{tabular}

Bayesian contingency analysis found evidence in favor of the null hypothesis regarding the strategies used by standard and causal participants who used a calculator in their solution, $\mathrm{BF}_{10}=0.16$. There was strong evidence however, of group differences in solution strategy for those who did not use a calculator, $\mathrm{BF}_{10}=21.33$. Again, we need to be cautious in interpreting these group differences because of the small cell sizes. Nevertheless, the table suggests that the standard and causal groups differed in their most favored solution strategies. In the standard group, the most common strategies were to report the given likelihood or other. In the causal group, the most common strategies were to report the given false positive rates or to calculate either the Bayesian numerator or denominator (without combining them).

A one-way BANOVA showed that there was weak evidence that the non-Bayesian strategies in Table 4 (i.e., excluding the normatively correct solution) used by the causal group produced lower probability estimates $(M=24 \%, S E M=4.70)$ than those used by the standard group $(M=39 \%, S E M=$ 4.90), $\mathrm{BF}_{10}=2.02$.
Bayesian contingency analysis found evidence against a relationship between numerical ability as measured by scores on the BNT and the type of non-Bayesian strategy employed, $\mathrm{BF}_{10}=0.02$. However, it is notable that four of the five causal group participants who successfully calculated the Bayesian numerator or denominator without the aid of a calculator achieved the maximum BNT score.

Like other recent work (e.g., Cohen \& Staub, 2015), our process tracing results highlight the diversity of problemsolving strategies adopted by different individuals on Bayesian reasoning tasks. Within each group, individuals differed markedly in the extent to which they considered the various problem components and combined these to arrive at a numerical estimate.

Nevertheless, the data suggest some important differences in reasoning strategy between the standard and causal groups. Those in the standard group often used strategies that centered around the likelihood $p(\mathrm{D} \mid \mathrm{H})$. The provision of causal information about false positives produced two notable shifts in strategy; more participants in the causal condition reported the false positive rate as their answer, whereas others

Table 4 Experiment 3 Frequency (and within-group proportion) of specific solution strategies

\begin{tabular}{|c|c|c|c|c|c|c|c|c|}
\hline Condition & $\begin{array}{l}\text { Reports } \\
\text { base } \\
\text { rate }(0.01)\end{array}$ & $\begin{array}{l}\text { Reports } \\
\text { likelihood } \\
(0.8)\end{array}$ & $\begin{array}{l}\text { Reports false } \\
\text { positive rate } \\
(0.15 / 0.3 / \\
0.5)^{\mathrm{a}}\end{array}$ & $\begin{array}{l}p(\mathrm{D} \mid \mathrm{H})- \\
p(\mathrm{D} \mid \neg \mathrm{H}) \\
(0.8-0.15)\end{array}$ & $\begin{array}{l}p(\mathrm{D} \mid \mathrm{H}) * \\
p(\mathrm{D} \mid \neg \mathrm{H}) \\
(0.8 * 0.15)\end{array}$ & $\begin{array}{l}\text { Bayes rule numerator } \\
\text { or denominator } \\
(0.8 * 0.01) \text { or }((0.8 * \\
0.01)+(0.99 * 0.15))\end{array}$ & $\begin{array}{l}\text { Normative } \\
\text { solution }\end{array}$ & Other \\
\hline $\begin{array}{l}\text { Standard/No } \\
\quad \text { calculator } \\
(n=17)\end{array}$ & $2(11.8 \%)$ & $6(35.3 \%)$ & 0 & $3(17.6 \%)$ & 0 & 0 & 0 & $6(35.3 \%)$ \\
\hline $\begin{array}{l}\text { Causal/No } \\
\quad \text { calculator } \\
(n=25)\end{array}$ & $4(16.0 \%)$ & $3(12.0 \%)$ & $6(24.0 \%)$ & $1(4.0 \%)$ & $2(8.0 \%)$ & $5(20.0 \%)$ & 0 & $4(16.0 \%)$ \\
\hline $\begin{array}{l}\text { Standard/Calculator } \\
\quad(n=23)\end{array}$ & 0 & 0 & $1(4.3 \%)$ & $1(4.3 \%)$ & $1(4.3 \%)$ & $8(34.8 \%)$ & $5(21.7 \%)$ & $7(30.4 \%)$ \\
\hline $\begin{array}{l}\text { Causal/Calculator } \\
\quad(n=15)\end{array}$ & $1(6.7 \%)$ & 0 & $1(6.7 \%)$ & $1(6.7 \%)$ & $2(13.3 \%)$ & $4(26.7 \%)$ & $2(13.3 \%)$ & $4(26.7 \%)$ \\
\hline Total & $7(8.8 \%)$ & $9(11.3 \%)$ & $8(10.0 \%)$ & $6(7.5 \%)$ & $5(6.3 \%)$ & $17(21.3 \%)$ & $7(8.8 \%)$ & $21(26.3 \%)$ \\
\hline
\end{tabular}

${ }^{\text {a }}$ Note that this column includes causal participants who reported both components of the false positive rate (i.e., reported 0.3 and 0.5 ). The majority in the causal group (six out of seven) did so 
successfully computed the numerator or denominator of the Bayesian solution.

An unexpected finding was that the effects of causal information on statistical judgments were moderated by calculator use. Suggestive effects of causal information on solution strategies for the mammogram problem were only noted in the subset of participants who did not use a calculator. Those who used a calculator gave probability estimates that were closer to the normative solution and their solution strategies were unaffected by the provision of causal information. Previous work examining intuitive statistical estimation has generally neglected calculator use as a variable that could moderate solution strategies. Such work has either failed to distinguish between participants who did or did not use a calculator (e.g., Gigerenzer \& Hoffrage, 1995; Krynski \& Tenenbaum, 2007) or has discouraged calculator use in order to examine intuitive solution strategies (e.g., Cohen \& Staub, 2015; McNair \& Feeney, 2014, 2015). Our work shows that calculator use is an important moderator, which should be given greater consideration in future work on intuitive statistical judgment. If the primary interest is in examining the impact of causal information on intuitive solutions, our findings suggest that calculator use should be prevented or explicitly manipulated between groups.

It is hard to know exactly what influenced our participants' decisions about whether to use a calculator. It may be that those who used a calculator had a better understanding of the complexity of the mammogram problem and of the need to integrate the various statistical components to reach a solution. More generally, it may be that participants who used a calculator were those who were most highly motivated to perform well at the task or had a more reflective cognitive style (Frederick, 2005; Sirota et al., 2014) that led them to engage more deeply with the problem. This is consistent with work that shows that calculator use not only improves performance in mathematical problem solving but is associated with more positive attitudes towards mathematics (Ellington, 2006; Rakes, Valentine, McGatha, \& Ronau, 2010).

Combined data analysis As a final test of the weak facilitation hypothesis, we combined the probability estimate data from the conditions using percentage formats in each experiment (combined standard $n=139$, causal $n=129$ ). A BANOVA comparing open-ended estimates found very strong evidence that causal explanation $(M=26.28, S E M=$ 2.71) led to lower estimates that were closer to the normative response than did standard explanation $(M=41.27, S E M=$ 2.6), $\mathrm{BF}_{10}=225.62, \eta_{\mathrm{p}}{ }^{2}=0.06$. As a final test of the strong facilitation hypothesis, estimates were coded as correct, using the same criteria as the individual studies, or were coded as incorrect. A Bayesian contingency analysis found strong evidence in favor of the null hypothesis that there was no difference in the proportion of normatively correct responses between standard $(10.8 \%)$ and causal groups $(12.5 \%), \mathrm{BF}_{10}=$ 0.11 .

\section{General discussion}

The current studies reexamined claims that clarifying the causal origin of key statistics leads to improved performance on Bayesian statistical problems. Contrary to Krynski and Tenenbaum (2007), we found no evidence that causal explanation increases normative responding on such problems. Explaining the causal origin of false positives reduced the magnitude of errors in probability estimates, but this effect was limited to cases where both $p(-\mathrm{H})$ and $p(\mathrm{D} \mid-\mathrm{H})$ were presented and was only robust when percentage formats were used (Experiment 2).

Uncovering the specific reasoning strategies that produce this weak causal facilitation was a key aim of our processtracing study. This revealed that facilitation was largely the result of a mixture of non-Bayesian strategies used by individuals in the causal condition. The non-Bayesian strategies used by those in the causal condition led to lower probability estimates than the non-Bayesian strategies used by participants in the standard condition.

Most often, the strategies used in the causal condition simply involved reporting the explained false positive statistics or a non-Bayesian combination of the false positive with other statistics. These patterns are similar in many respects to those noted in early attentional accounts of causal knowledge effects on base rate neglect (e.g., Ajzen, 1977; Bar-Hillel, 1980; Tversky \& Kahneman, 1980).

Explaining the cause of false positives in the mammogram problem did lead some participants to provide a closer approximation of the Bayesian solution (i.e., calculating the Bayes rule numerator or denominator). This appears to be more than a simple attentional effect - it suggests a more sophisticated heuristic in which causal explanation leads to an active search for ways of combining the false positive rate with other statistics. However, it is important to remember that the number of such cases was small and limited to those who did not use a calculator.

Just why individuals responded in different ways to causal explanation of false positives remains to be determined. One possibility, suggested by our data, is that those who have more mathematical aptitude and/or experience are likely to show the greatest benefit from causal explanation. After the causal explanation has highlighted the need to incorporate false positive information into a probability estimate, these participants were more successful in doing so in ways that are consistent with components of the normative solution. In this respect, our 
findings are consistent with McNair and Feeney (2015), who found that causal information facilitated performance only in highly numerate participants. However, our protocol results go further to show that causal explanation can prompt the use of other kinds of non-Bayesian strategies in people who may not have high levels of mathematical competence.

\section{Implications for the causal Bayesian approach}

Krynski and Tenenbaum (2007) argue that providing a generative cause for false positives leads to better mapping of false positive statistics to the components of intuitive causal models. This, in turn, promotes the normative use of false positives in deriving probability estimates. Our protocol data provide only weak support for the latter claim. In no case did causal explanation of the relevant statistics lead to more normative responding. Causal information did affect probability judgments, but primarily by drawing attention to the explained statistics or, in a minority of cases, leading people to calculate one component of the normative solution.

The more general claim that causal explanation can alter people's mental models of intuitive probability problems was supported, but only in a very general way. Causal explanation did prompt people to incorporate false positive statistics into their problem solutions. When their causal origin was unknown, such statistics were often neglected. Our process-tracing analysis, however, highlights the individual variability in causal models of judgment problems. Moreover, in many cases, the new mental models that were prompted by additional causal explanation appeared to be no more sophisticated or normative than those in the standard condition.

Our results do not mean that we should abandon the causal Bayesian approach. In future research, however, we see a detailed investigation of the way that causal explanations shape mental representations of statistical problems as a more worthwhile goal than searching for evidence (or lack thereof) for causal facilitation of performance. There is also a need to examine how the effects of causal information may change as values of parameters such as the base rate, likelihoods, and false-positive rate are varied (cf. Cohen \& Staub, 2015). Both the current work and previous studies (Hayes et al., 2014; Rottman \& Hastie, 2014; Waldmann, 2007) show that causal information can alter people's interpretation of the components of problems involving intuitive statistical estimation. However only when such change in problem representation is accompanied by mathematical expertise are we likely to see any improvement in normative accuracy.
Author note This work was supported by Australian Research Council Discovery Grant DP120100266 to the first and fourth authors and a Future Fellowship FT110100151 to the fourth author. We thank Rachel Lodge and Kelly Jones for help with programming and running the experiments and Natali Dilevski for assistance in manuscript preparation.

\section{Appendix A}

\section{Normative solutions}

1. Mammogram problem

The normative probability of cancer given a positive mammogram is given by:

$$
\begin{aligned}
p(H \mid D) & =\frac{p(H) \times(D \mid H)}{p(H) \times p(D \mid H)+p(\neg H) \times p(D \mid \neg H)} \\
& =\frac{0.01 \times 0.80}{(0.01 \times 0.80)+(0.99 \times 0.15)} \\
& \approx 0.051
\end{aligned}
$$

2. Drug problem

$$
\begin{aligned}
p(H \mid D) & =\frac{p(H) \times p(D \mid H)}{p(H) \times p(D \mid H)+p(\neg H) \times p(D \mid \neg H)} \\
& =\frac{0.04 \times 0.75}{(0.04 \times 0.75)+(0.96 \times 0.10)} \\
& \approx 0.242
\end{aligned}
$$

\section{Appendix B}

\section{Experiment 1: Mammogram (mixed format)}

The following statistics are known about women at age 60 who participate in a routine mammogram screening for breast cancer:

1 in every 100 women has breast cancer at the time of screening.

8 in every 10 of those with breast cancer receive a positive mammogram.

15 in every 100 women without breast cancer will get a positive mammogram. [Standard]

30 in every 100 women have a benign cyst at the time of screening. Of those with a benign cyst, half will get a positive mammogram. [Causal]

Suppose a woman gets a positive result during a routine mammogram screening. Without knowing any other symptoms, what is the probability that she has breast cancer? 


\section{Experiment 1. Drug-test problem (percentage format)}

An airline company is concerned to make sure that its workforce is drug free. To ensure a thorough screening for illegal drug use, the company has adopted a policy where all employees are required to supply a urine sample.

From company records, it is known that:

4 percent of employees are known to use illegal drugs.

The following is known about screening of a single urine sample:

Of those who use illegal drugs, $75 \%$ will receive a positive result on the urine test.

Of those who do not use illegal drugs, $10 \%$ will receive a positive result on the urine test. [Standard]

20 percent of employees are taking legal over-the-counter medications. Of those taking legal medications, chemicals in the medications will trigger a positive result on the urine test $50 \%$ of the time. [Causal]

Suppose an employee gets a positive result on a urine sample. Without knowing any other information, what are the chances that the employee is using illegal drugs?

\section{Experiment 1. Drug test (frequency format)}

An airline company is concerned to make sure that its workforce is drug free. To ensure a thorough screening for illegal drug use the company has adopted a policy where all employees are required to supply a urine sample.

From company records:

4 out every 100 employees are known to use illegal drugs.

The following is known about screening of a single urine sample:

Of those who use illegal drugs, 15 in every 20 will receive a positive result on the urine test.

Of those who do not use illegal drugs, 10 in every 100 will receive a positive result on the urine test. [Standard]

20 in every 100 employees are taking legal over-thecounter medications. Of those taking legal medications, chemicals in the medications will trigger a positive result on the urine test half of the time. [Causal]

Imagine that we have selected a random sample of $100 \mathrm{em}-$ ployees at the airline company. Without knowing any other information, on average, how many people who receive a positive result on the urine test do you expect will be taking illegal drugs?

\section{Experiment 1. Drug-test (mixed format)}

An airline company is concerned to make sure that its workforce is drug free. To ensure a thorough screening for illegal drug use the company has adopted a policy where all employees are required to supply a urine sample.

From company records:

4 out every 100 employees are known to use illegal drugs.
The following is known about screening of a single urine sample:

Of those who use illegal drugs, 15 in every 20 will receive a positive result on the urine test.

Of those who do not use illegal drugs, 10 in every 100 will receive a positive result on the urine test. [Standard]

20 in every 100 employees are taking legal over-thecounter medications. Of those taking legal medications, chemicals in the medications will trigger a positive result on the urine test half of the time. All others will receive a negative urine result. [Causal]

Suppose an employee gets a positive result on a urine sample. Without knowing any other information, what are the chances that the employee is using illegal drugs?

\section{Appendix C}

\section{Experiment 3: Process tracing instructions}

\section{General introduction}

This experiment looks at how people solve problems. An important part of this experiment is for you to think aloud when I give you a problem to solve. I want you to focus on the task and say whatever you are thinking as you try to solve the problem. Please tell me as much as possible about what you are thinking — don't hold anything back.

The session will be recorded to make sure I get all of your thoughts.

\section{Think-aloud modeling}

To give you a better idea of what I want you to do, I will now show you a short video clip of someone thinking aloud while solving an anagram. Here is the anagram the person is trying to solve. [Think-aloud modeling: clip of someone trying to solve the anagram for 'ARMEK' while thinking aloud]

\section{Think-aloud practice}

So maker was the correct answer. She solved that problem fairly quickly. Some of the problems you see may be a bit more difficult, but don't worry, just take your time. Now I am going to give you a chance to have a couple of practices before the main task. I'm going to ask you to solve another anagram and to think aloud while you are solving it. You can write down any working out on this sheet of paper as you go, but please remember to tell me what you are thinking while you work things out.

[Think Aloud Practice 1: Anagram ARNTI] (4 minute timeout: If you can't think of the correct answer, that's okay. The correct answer is TRAIN. Let's move on to the next task. 
That was good. So now we'll do one more practice item. Please remember to tell me everything that comes into your head while you are thinking. Now, try to solve this problem. [Think Aloud Practice 2: multiplication without a calculator: $36 \times 24=?]$

\section{Main task}

Okay, now we'll move onto the main task. I'm going to give you a different type of problem that involves some percentages. Remember to think aloud while you solve it. I won't be able to answer any questions about the task while you are working it out, so just do whatever you think is best. What we're really interested in is how people approach the problem rather than whether or not they come up with the right answer, so take as much time as you like. Do you have any questions?

Okay, here is the problem. Please read the problem aloud and make any comments you like. Once you have finished reading the problem, please attempt to answer it and rate your confidence in your answer on the sheet. Remember to tell me your thoughts as they come to mind. You may also use a calculator and write down any working out on the question paper. But please remember to tell me what you are thinking while you work things out. [Present mammogram task]

General prompts (presented during the solution process): Keep going, you are doing really well. Just remember to keep talking and telling me what you are thinking.

End prompt: Okay, so, do you have anything else to add? Okay, now I will stop the recording.

\section{References}

Ajzen, I. (1977). Intuitive theories of events and the effects of base-rate information on prediction. Journal of Personality and Social Psychology, 35, 303-314. https://doi.org/10.1037/ 0022-3514.35.5.303

Barbey, A. K., \& Sloman, S. A. (2007). Base-rate respect: From ecological rationality to dual processes. Behavioral and Brain Sciences, 30, 241-254. https://doi.org/10.1017/S0140525X07001653

Bar-Hillel, M. (1980). The base-rate fallacy in probability judgments. Acta Psychologica, 44, 211-233. doi:https://doi.org/10.1016/00016918(80)90046-3

Beyth-Marom, R., \& Fischhoff, B. (1983). Diagnosticity and pseudodiagnosticity. Journal of Personality and Social Psychology, 45, 1185-1195. https://doi.org/10.1037/0022-3514.45.6.1185

Bröder, A. (2003). Decision making with the "adaptive toolbox": Influence of environmental structure, intelligence, and working memory load. Journal of Experimental Psychology: Learning, Memory, and Cognition, 29, 611-625. https://doi.org/10.1037/ 0278-7393.29.4.611

Cohen, A. L., \& Staub, A. (2015). Within-subject consistency and between-subject variability in Bayesian reasoning strategies. Cognitive Psychology, 81, 26-47. https://doi.org/10.1016/j. cogpsych.2015.08.001
Cokely, E. T., Galesic, M., Schulz, E., Ghazal, S., \& Garcia-Retamero, R. (2012). Measuring risk literacy: The Berlin numeracy test. Judgment and Decision Making, 7, 25-47.

Cokely, E. T., \& Kelley, C. M. (2009). Cognitive abilities and superior decision making under risk: A protocol analysis and process model evaluation. Judgment and Decision Making, 4, 20-33.

Cosmides, L., \& Tooby, J. (1996). Are humans good intuitive statisticians after all? Rethinking some conclusions from the literature on judgment under uncertainty. Cognition, 58, 173. https://doi.org/10.1016/0010-0277(95)00664-8

Ellington, A. J. (2006). The effects of nan-CAS graphing calculators on student achievement and attitude levels in mathematics: A metaanalysis. School Science and Mathematics, 106, 16-26.

Evans, J. S. B., Handley, S. J., Perham, N., Over, D. E., \& Thompson, V. A. (2000). Frequency versus probability formats in statistical word problems. Cognition, 77, 197-213. https://doi.org/10.1016/S0010-0277(00)00098-6

Fernbach, P. M., Darlow, A., \& Sloman, S. A. (2011). Asymmetries in predictive and diagnostic reasoning. Journal of Experimental Psychology: General, 140, 168-185. https://oi.org/10.1037/a0022100

Fiedler, K., Brinkmann, B., Betsch, T., \& Wild, B. (2000). A sampling approach to biases in conditional probability formats: Beyond base rate neglect and statistical format. Journal of Experimental Psychology: General, 129, 399-418.

Frederick, S. (2005). Cognitive reflection and decision-making. Journal of Economic Perspectives, 19, 25-42. https://doi.org/10.1257/ 089533005775196732

Gigerenzer, G., \& Hoffrage, U. (1995). How to improve Bayesian reasoning without instruction: Frequency formats. Psychological Review, 102, 684-704. https://doi.org/10.1037/0033-295X.102.4.684

Hagmayer, Y., \& Sloman, S. A. (2009). Decision makers conceive of their choices as interventions. Journal of Experimental Psychology: General, 138, 22-38. https://doi.org/10.1037/a0014585

Hawkins, G. E., Hayes, B. K., Donkin, C., Pasqualino, M., \& Newell, B. R. (2015). A Bayesian latent mixture model analysis shows that informative samples reduce base rate neglect. Decision, 2, 306318. https://doi.org/10.1037/dec0000024

Hayes, B. K., Hawkins, G. E., \& Newell, B. R. (2016). Consider the alternative: The effects of causal knowledge on representing and using alternative hypotheses in judgments under uncertainty. Journal of Experimental Psychology: Learning, Memory and Cognition, 42, 723-739. https://doi.org/10.1037/xlm0000205

Hayes, B. K., Hawkins, G. E., Newell, B. R., Pasqualino, M., \& Rehder, B. (2014). The role of causal models in multiple judgments under uncertainty. Cognition, 133, 611-620. https://doi.org/10.1016/j. cognition.2014.08.011

Hayes, B. K., \& Rehder, B. (2012). The development of causal categorization. Cognitive Science, 36, 1102-1128. https://doi. org/10.1111/j.1551-6709.2012.01244.x

Hill, W. T., \& Brase, G. L. (2012). When and for whom do frequencies facilitate performance? On the role of numerical literacy. The Quarterly Journal of Experimental Psychology, 65, 2343-2368. https://doi.org/10.1080/17470218.2012.687004

Jamil, T.L., Morey, R. D., Love, J., Marsman, M., \& Wagenmakers, E. J. (2017). Default "Gunel and Dickey" Bayes factors for contingency tables. Behavior Research Methods, 49, 638652. https://doi.org/10.3758/s13428-016-0739-8

Kass, R. E., \& Raftery, A. E. (1995). Bayes factors. Journal of the American Statistical Association, 90, 773-795.

Krynski, T. R., \& Tenenbaum, J. B. (2007). The role of causality in judgment under uncertainty. Journal of Experimental Psychology: General, 136, 430-450. https://doi.org/10.1037/0096-3445.136.3.430

Lesage, E.N, \& De Neys, W. (2013). Evolutionary modules and Bayesian facilitation: The role of general cognitive resources. Thinking and Reasoning, 19, 27-53. 
Levin, I. P., \& Jasper, J. D. (1995). Phased narrowing: A new process tracing method for decision-making. Organizational Behavior and Human Decision Processes, 64, 1-8. https://doi.org/10.1006/obhd.1995.1084

Love, J., Selker, R., Marsman, M., Jamil, T., Dropmann, D., Verhagen, A. J., \& Wagenmakers, E.-J. (2016). JASP (Version 0.8) [Computer software]. Retrieved from http://jasp-stats.org

McNair, S., \& Feeney, A. (2014). When does information about causal structure improve statistical reasoning? The Quarterly Journal of Experimental Psychology, 67, 625-645. https://doi.org/10.1080/ 17470218.2013.821709

McNair, S., \& Feeney, A. (2015). Whose statistical reasoning is facilitated by a causal structure intervention? Psychonomic Bulletin \& Review, 22, 258-264. https://doi.org/10.3758/s13423-014-0645-y

Rakes, C. R., Valentine, J. C., McGatha, M. B., \& Ronau, R. N. (2010). Methods of instructional improvement in algebra: A systematic review and meta-analysis. Review of Educational Research, 80, 372-400.

Reyna, V. F., Nelson, W. L., Han, P. K., \& Dieckmann, N. F. (2009). How numeracy influences risk comprehension and medical decision-making. Psychological Bulletin, 135, 943973. https://doi.org/10.1037/a0017327

Rouder, J. N., Morey, R. D., Verhagen, J., Swagman, A. R., \& Wagenmakers, E. J. (2016). Bayesian analysis of factorial designs. Psychological Methods. Advance online publication. https://doi.org/10.1037/met0000057
Rottman, B. M., \& Hastie, R. (2014). Reasoning about causal relationships: Inferences on causal networks. Psychological Bulletin, 140, 109-139. https://doi.org/10.1037/a0031903

Sirota, M., Juanchich, M., \& Hagmayer, Y. (2014). Ecological rationality or nested sets? Individual differences in cognitive processing predict Bayesian reasoning. Psychonomic Bulletin \& Review, 21, 198-204. https://doi.org/10.3758/s13423-013-0464-6

Sloman, S. A., Over, D., Slovak, L., \& Stibel, J. M. (2003). Frequency illusions and other fallacies. Organizational Behavior and Human Decision Processes, 91, 296-309. https://doi.org/10.1016/S07495978(03)00021-9

Tversky, A., \& Kahneman, D. (1974). Judgment under uncertainty: Heuristics and biases. Science, 185, 1124-1131. https://doi.org/10. 1126/science.185.4157.1124

Tversky, A., \& Kahneman, D. (1980). Causal schemas in judgments under uncertainty. In M. Fishbein (Ed.), Progress in social psychology (pp. 49-72). Hillsdale, NJ: Erlbaum.

Waldmann, M. R. (2007). Combining versus analyzing multiple causes: How domain assumptions and task context affect integration rules. Cognitive Science, 31, 233-256. https://doi.org/10.1080/ 15326900701221231

Waldmann, M. R., Hagmayer, Y., \& Blaisdell, A. P. (2006). Beyond the information given causal models in learning and reasoning. Current Directions in Psychological Science, 15, 307-311. https://doi.org/ 10.1111/j.1467-8721.2006.00458.x 